\title{
Prospective practice survey of management of cetuximab-related skin reactions
}

\author{
R. T. Lugtenberg ${ }^{1}$ - C. B. Boers-Doets ${ }^{2,3}$ • P. O. Witteveen ${ }^{4}$ - C. M. L. van Herpen ${ }^{5}$ - A. N. M. Wymenga ${ }^{6}$. \\ J. W. B. de Groot ${ }^{7}$ - A. Hoeben ${ }^{8}$ • C. del Grande ${ }^{9}$ • B. van Doorn ${ }^{9}$ • J. J. Koldenhof ${ }^{4}$ • C. M. L. Driessen ${ }^{5} \cdot$ H. Gelderblom ${ }^{1}$
}

Received: 8 September 2020 / Accepted: 26 October 2020 / Published online: 4 November 2020

(C) The Author(s) 2020

\begin{abstract}
Purpose Evidence-based guidelines on how to prevent or treat cetuximab-related skin reactions are lacking and multiple care and management strategies are used. The main purpose of the present study is to gain information about the different skincare products being used against skin reactions in metastatic colorectal cancer (mCRC) and recurrent/ metastatic (R/M) or locally advanced (LA) squamous cell cancer of the head and neck (SCCHN) patients treated with cetuximab.

Methods An open-label, prospective observational study conducted in the Netherlands. The occurrence of skin reactions and the care and management options taken were documented for 16 weeks, starting from the first administration of cetuximab.

Results A total of 103 patients were included in 7 hospitals. 38 patients (37\%) developed a grade $\geq 2$ skin reaction. Eighty-six patients could be analysed for the primary endpoint $(73.3 \%$ males, mean age 62.4 years, $n=44$ LA SCCHN, $n=16 \mathrm{R} / \mathrm{M}$ $\mathrm{SCCHN}, n=26 \mathrm{mCRC}$ ). The most frequently used skin products at some point during the observation period were moisturizing products (70\%), systemic antibiotics (64\%), topical antibiotics (58\%), lipid-regenerating (28\%) and other topical products (28\%). The overall use of products gradually increased from baseline to week $6-10$, reducing by week 16 . Hospital protocols were the primary reason $(>50 \%)$ for choice of the skincare products and medications.

Conclusion A variety of skin care products and antibiotics were commonly used. Only few patients developed severe cutaneous reactions. For patients, the occurrence of skin reactions did not influence their willingness to continue cetuximab therapy.
\end{abstract}

Keywords Cetuximab $\cdot$ Skin reaction $\cdot$ Management $\cdot$ Head and neck cancer $\cdot$ Colorectal cancer

\section{Introduction}

Cetuximab is a chimeric monoclonal antibody that binds and inactivates the epidermal growth factor receptor (EGFR). As a consequence of this blockade, extracellular

R. T. Lugtenberg

r.t.lugtenberg@lumc.nl

1 Department of Medical Oncology, Leiden University Medical Center, Albinusdreef 2, P.O. Box 9600, 2300

RC Leiden, The Netherlands

2 Department of Medical Strategy, CancerMed, Wormer, The Netherlands

3 Department of Adverse Event Research \& Valorisation, Impaqtt Foundation, Wormer, The Netherlands

4 Department of Medical Oncology, University Medical Center Utrecht, Utrecht, The Netherlands activators like the epidermal growth factor (EGF) cannot bind to the receptor anymore and tumour-promoting downstream signalling cascades cannot be activated. This mechanism of blocking EGFR is an important strategy in the treatment of metastatic colorectal cancer (mCRC) and

5 Department of Medical Oncology, Radboud University Nijmegen Medical Center, Nijmegen, The Netherlands

6 Department of Medical Oncolog, Medical Spectrum Twente, Enschede, The Netherlands

7 Department of Medical Oncology, Isala Oncology Center, Zwolle, The Netherlands

8 Department of Medical Oncology, School for Oncology and Developmental Biology (GROW), Maastricht University Medical Center+, Maastricht, The Netherlands

9 Merck B.V., Netherlands, an affiliate of Merck KgaA, Darmstadt Germany, Schiphol-Rijk, The Netherlands 
squamous cell cancer of the head and neck (SCCHN) [1-4].

However, EGFR is expressed not solely on tumour cells but also on cells of the epidermis. There, EGF/EGFRmediated signalling cascades regulate physiologic homeostasis of the tissue. Inhibition of EGFR causes abnormal growth and migration behaviour of keratinocytes. It also changes keratinocyte differentiation and maturation. These changes are accompanied by an inflammatory state of the dermis [5, 6]. In light of the physiologic relevance of the EGFR signalling cascade, one side effect of this type of drug can be explained: various cutaneous toxicities.

Most patients receiving cetuximab develop skin reactions. In the majority of cases, skin reactions like xerosis, maculo-papular rash, papulo-pustular rash, pruritus or fissures occur within the first weeks of therapy and the occurrence is time-dependent. The most frequently reported events are papulo-pustular rash (80\%) which affects predominantly the face, upper trunk and the scalp. Skin reactions caused by cetuximab can severely affect patient health-related quality of life (HRQoL) and can lead eventually to dose delays, dose reductions and even permanent discontinuation of treatment $[7,8]$. Normally, skin reactions resolve once the therapy is stopped or following dose reductions. Cetuximab-induced skin reactions have been positively associated with better treatment response and longer survival in mCRC and R/M SCCHN [9-11]. Therefore, finding the optimal strategies to prevent, recognize early and treat skin reactions seems necessary. During cetuximab therapy multiple care and management options to reduce the severity of the skin reactions are used. Secondary to general measures like the avoidance of intense sun exposure and the use of disinfectant synthetic detergents and proper skin care is recommended. However, most recommendations on how to prevent or treat skin reactions are based on expert opinions, since evidence-based guidelines are scarce [12]. Optimal strategies for the management of cetuximab-induced skin reactions remain unclear. Besides the prophylactic use of emollients, use of oral or topical tetracyclines and topical application of $1 \%$ hydrocortisone cream together with a moisturizer can be considered to reduce the severity of skin reactions [13, 14], or other topical agents such as vitamin $\mathrm{K}_{1}$ cream and antihistamines in case of pruritus $[15,16]$. As cetuximab is increasingly being used in cancer therapy, optimal strategy to detect and treat antiEGFR-induced skin reactions can help clinicians to improve patient care.

The primary objective of this current practice survey is to gain information about the perceived effectiveness of the measures taken against the skin reactions in $\mathrm{mCRC}$ and SCCHN patients treated with cetuximab in Dutch daily practice.

\section{Methods}

\section{Study design}

We performed a prospective observational study with a nonexperimental cohort design. The primary objective of the study was to provide insight into the use of the different applied prophylactic and reactive skincare products used in patients with cetuximab-related skin reactions. Secondary objectives were incidence and grading of skin reactions as measured by NCI-CTCAE v4.03: reasons for premature discontinuation of cetuximab treatment; reasons for choice of applied skin products; the percentage of days under skin care products during the observation period, HRQoL as measured by the FACT-EGFRI-18; the assessment of the perceived effectiveness of the applied skin products by physicians, nurses and patients.

\section{Patients}

15 to 20 centres within the Netherlands that treat metastatic colorectal cancer (mCRC) and/or recurrent, metastatic or locally advanced squamous cell cancer of head and neck (SCCHN) patients with cetuximab were planned to include 100 patients. Cetuximab treatment consisted of monotherapy or in combination with radiation therapy or chemotherapy. Eligible patients were $\geq 18$ years of age, with a histologically proven SCCHN or RAS wild type mCRC who were planned for cetuximab treatment; a wash-out period of 3 months for previous treatment with cetuximab; an Eastern Cooperative Oncology Group (ECOG) performance status $\leq 2$; absence of active skin reactions/infections for which use of any topical treatment was needed; absence of the presence of a skin condition in the face, neck or chest that may obscure skin reactions to cetuximab (e.g. excessive facial hair, excessive scarring, sunburn or other disfigurements); and written informed consent.

\section{Data collection}

From the first administration of cetuximab (baseline visit) and during follow-up visits the healthcare professional (treating physician or (research) nurses), using online eCRF, evaluated and assessed skin reactions, skin care management and other patient characteristics. Follow-up visits were scheduled for mCRC and R/M SCCHN patients at weeks 2, 4, 6, 10 and 16 , and for LA SCCHN patients at weeks $2,4,6$ and 8-10, since these patients only receive cetuximab for 8 weeks. Skin reactions were assessed as measured by the National Cancer Institute's Common Terminology Criteria for Adverse Events (NCI-CTCAE) v4.03. HRQoL was assessed with the FACTEGFRI-18, a questionnaire developed to assess HRQoL related to dermatologic reactions from EGFRI treatment and were 
completed by patients before every follow-up visit $[17,18]$. The FACT-EGFRI-18 is an 18-item Likert-scaled questionnaire, with response scores ranged from 0 to 4 . Product-specific, perceived effectiveness was assessed by using 5 point scales and were used for both skin care products (e.g. moisturizers, lipid-regenerating products, antiseptic products, ureacontaining products, vitamin $\mathrm{K}_{1}$ cream and others ) and pharmacological agents (topical or oral antibiotics, local anaesthetics antihistamines, topical glucocorticosteroids or others).

\section{Therapeutic plan}

Cetuximab was administered once a week intravenously, in an initial loading dose of $400 \mathrm{mg}$ per $\mathrm{m}^{2}$ body surface area, followed by doses of $250 \mathrm{mg}$ per $\mathrm{m}^{2}$. Patients received premedication with an antihistamine and in some cases also a corticosteroid.

\section{Statistical analysis}

Patients' medical records and online electronic case report forms (eCRFs) were used for primary data sources. The full analysis set (FAS) included all enrolled patients who had sufficient documentation of cetuximab treatment and sufficient data for the primary endpoint at least at baseline. The safety analysis set (SAS) included all enrolled patients for whom cetuximab treatment was started.

Descriptive statistical methods were used for all variables (e.g. mean, standard deviation, median, interquartile range for continuous variables and proportions for categorical variables). In addition, 95\% confidence intervals were calculated for point estimates (mean, median or proportion). Statistical analysis was performed using Statistical Analysis System Version 9.1.3 (NC, USA).

\section{Results}

\section{Patients}

Between September 2013 and December 2016, 103 patients from 7 Dutch centres were enrolled in the study. One of the 103 patients was excluded from the Safety Analysis Set (SAS) because the patient withdrew from the study prior to start of treatment (Fig. 1). Eighty-six out of the 103 patients (83.5\%) were included in the full analysis set (FAS). Reasons for 17 patients being excluded from the FAS were missing data: no clear diagnosis of mCRC or R/M SCCHN recorded $(n=4)$; no documented cetuximab treatment $(n=14)$ and insufficient data regarding the primary endpoint at baseline $(n=1)$. For some subjects, there was more than one reason for exclusion. Patients' demographic characteristics from the FAS are summarized in Table 1. Most patients were male (73\%) with a median age of 62 years (range 31-80). Cetuximab was administered in the majority of patients for locally advanced SCCHN $(n=44,51 \%)$ as primary treatment, with a planned treatment period of 8 weeks. 26 patients were treated for $\mathrm{mCRC}(30 \%)$ and 16 patients received cetuximab for $\mathrm{R} / \mathrm{M}$ SCCHN (19\%).

\section{Skin reactions incidence and grading}

89 of 102 patients $(87 \%)$ developed a skin reaction at some point during the observation period. Table 2 lists the incidence and severity of the skin reactions that occurred. The majority of these reactions were grade 1 or 2 (45 and 37\% respectively). Xerosis was reported in 68 patients (67\%), maculo-papular rash in $55(54 \%)$, papulo-pustular rash or acneiform eruption in $54(53 \%)$ and pruritus in $34(33 \%)$ patients. Less often observed reactions were paronychia, hand-foot skin reaction and acneiform rash.

\section{Premature discontinuations}

70 of 102 patients $(69 \%)$ discontinued participation in this study before 16 weeks. Main reasons were a planned treatment period of cetuximab treatment of 8 weeks in patients with locally advanced SCCHN (44 patients); disease progression (12 patients); death (2 patients); lost to follow-up (1 patient); or other reasons (10 patients). Only one patient discontinued prematurely due to skin reactions.

\section{Skin products used}

A summary of the use of the different applied skin care products and medications administered to prevent or treat skin reactions is provided per visit in Table 3 . The results of week 16 reflect on the patients who were still treated with cetuximab at that timepoint and were therefore mainly mCRC patients. At baseline, the most frequently used skin care products were moisturizing products (38\%), systemic antibiotics (20\%), lipid-regenerating products (12\%), vitamin $\mathrm{K}_{1}$ cream (9\%) and topical antibiotics $(6 \%)$. None of the patients used topical steroids, antiseptic products or urea-containing products at baseline. During the observation period of the study, the most frequently used skin products were moisturizing products $(70 \%)$, systemic antibiotics (64\%), topical antibiotics (58\%), lipid-regenerating products (28\%) and other topical treatments (28\%). The overall use of products showed a gradual increase from baseline to week $6 / 10$ for most of the products, reducing by week 16 . The overall use of topical antibiotics increased from baseline $(6 \%)$ to week $6(51 \%)$ reducing by week 16 $(31 \%)$. The overall use of systemic antibiotics increased from baseline (20\%) to week 4 (48\%) reducing by week $16(28 \%)$. The overall use of other topical treatments gradually increased from week $2(8 \%)$ to week 10 (23\%) reducing by week 16 


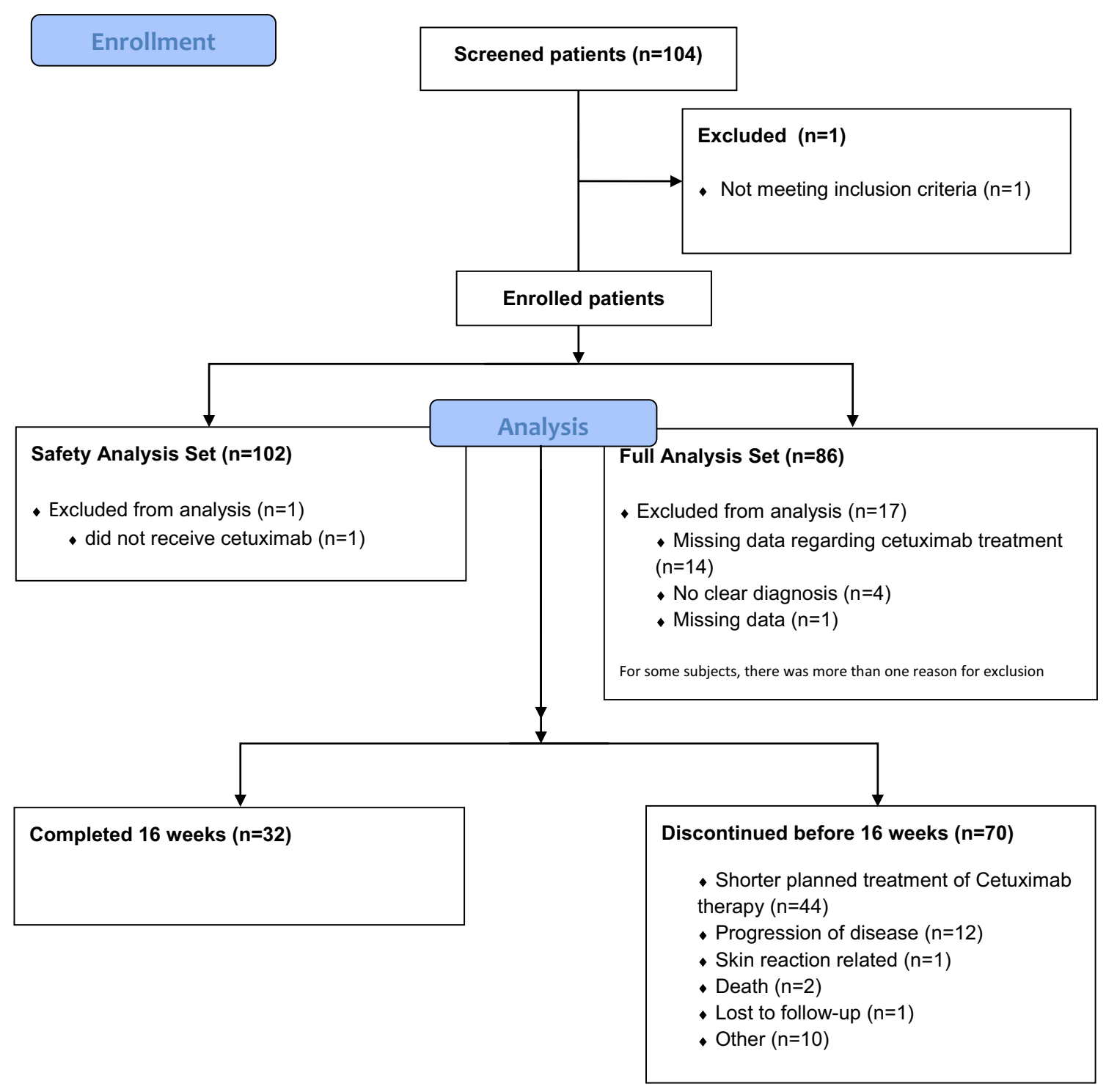

Fig. 1 Consort flow diagram

$(10 \%)$. The overall use of moisturizing products increased from baseline (38\%) to week $6(51 \%)$ reducing by week 16 $(35 \%)$. The overall use of lipid-generating products was identical at baseline and week $2(12 \%)$ and increased by week 6 $(26 \%)$ reducing gradually by week $16(3 \%)$. The overall use of vitamin $\mathrm{K}_{1}$ cream increased from baseline (9\%) to week 4 $(48 \%)$ reducing by week $16(28 \%)$. Other medications and skin care products like antihistamines, antiseptic and ureacontaining products were used in less than $15 \%$ of patients. Wet wraps were not used.

\section{Reasons for the choice of the applied skin care products}

The hospital protocol was the primary reason for choice of the skin care products at baseline (58\%) and during treatment (up to $71 \%$ ). Remaining reasons were investigator choice, previous experience, advice by a dermatologist or other reasons $(<20 \%$ each).

\section{Percentage of days under skin care products}

The proportion of days on skin treatment during the observation period (from the day of the first cetuximab treatment until 2-4 weeks after the last administration of cetuximab) was in a similar range for the specific type of treatments (about 50 to $70 \%)$.

\section{Health-related quality of life}

A continuous decrease in HRQoL, as measured by the FACTEGFRI-18, was seen during the observation period, with the strongest deterioration at week 10 followed by a slight improvement at week 16 . Both the total and all domain scores 
of the FACT-EGFRI-18 decreased over time, indicating that the treatment that patients received in this study, consisting of cetuximab monotherapy, or combined with radiation therapy or chemotherapy, had effect on physical functioning and overall well-being.

\section{Perceived effectiveness of applied skin products}

For almost all products effectiveness ratings varied across patients and HCPs from "no effect", "weak" and "moderate/strong", while "very strong" was rarely mentioned. During the observation period for all products, an increase in the percentage of patients who perceived at least weak efficacy was observed. Moderate to strong effectiveness was perceived most often by patients using moisturizing products (up to $90 \%$ at week 16); vitamin K cream (up to $78 \%$ at week 6); systemic antibiotics (up to $63 \%$ at week 16); topical antibiotics (up to $50 \%$ in weeks 6-16); and lipidregenerating products (up to $50 \%$ from week 2 and beyond). The HCPs' perceived effectiveness of the applied skin products is summarized in Fig. 2. In general, the percentages of the response "no effect" declined while "moderate" gained percentages over time.

\section{Mean HCPs' perceived effectiveness of different agents on skin reactions across visits}

The average numerical effectiveness values (from $0=$ no, to $4=$ very strong) of the assessments across all visits for each patient and type of medication were analysed with calculation of the mean \pm standard deviation (SD). Mean perceived effectiveness by HCPs (regardless of prophylactic or reactive usage) was highest for vitamin $\mathrm{K}_{1}$ cream (1.32 \pm 0.736 in 14 patients), lipid-regenerating product (1.05 \pm 0.752 in 24 patients) and systemic antibiotics $(1.05 \pm 1.066$ in 55 patients). The treatments with the lowest values were topical antibiotics ( $0.96 \pm 0.925$ in 50 patients), moisturizing products $(0.92 \pm 0.826$ in 60 patients) and other topical treatments $(0.85 \pm 0.887$ in 24 patients). The perceived effectiveness of prophylactic treatment was $0.90 \pm 1.025$ for moisturizing products in 37 patients and $0.26 \pm 0.554$ for systemic antibiotics in 16 patients. The results in the reactive group hardly differed with mean perceived effectiveness ranging from $0.67 \pm 0.900$ to $1.14 \pm 1.232$ for the different skin products.

\section{Patients' impression of skin reactions}

The vast majority of patients reported "neutral" impact of skin reactions on daily life during the total study period (95.3\% in week 2, 96.2\% in week 6 and $93.1 \%$ in week 16). Only 2 patients reported no impact of skin reactions on daily life. None of the patients reported "very strong" impact on daily life. Skin reactions did not influence the willingness
Table 1 Demographic characteristics and baseline data

\begin{tabular}{|c|c|}
\hline Characteristic & $(N=86)$ \\
\hline \multicolumn{2}{|l|}{ Sex, $n(\%)$} \\
\hline Male & $63(73.3)$ \\
\hline Female & $23(26.7)$ \\
\hline \multicolumn{2}{|l|}{ Age (years) } \\
\hline$n(\%)$ & $86(100)$ \\
\hline Mean \pm SD & $62.4 \pm 10.02$ \\
\hline Min; $\max$ & $31 ; 80$ \\
\hline \multicolumn{2}{|l|}{ Body surface area $\left(\mathrm{m}^{2}\right)$} \\
\hline$n(\%)$ & $86(100)$ \\
\hline Mean \pm SD & $1.92 \pm 0.24$ \\
\hline Min; $\max$ & $1.43 ; 2.73$ \\
\hline \multicolumn{2}{|l|}{ Diagnosis, $n(\%)$} \\
\hline $\mathrm{mCRC}$ & $26(30.2 \%)$ \\
\hline R/M SCCHN & $16(18.6 \%)$ \\
\hline LA SCCHN & $44(51.2 \%)$ \\
\hline \multicolumn{2}{|l|}{ ECOG performance score, $n(\%)$} \\
\hline 0 & $30(34.9 \%)$ \\
\hline 1 & $49(57.0 \%)$ \\
\hline 2 & $7(8.1 \%)$ \\
\hline \multicolumn{2}{|l|}{ Previous anticancer treatment, $n(\%)$} \\
\hline Surgery & $22(25.6)$ \\
\hline Chemotherapy & $29(33.7)$ \\
\hline Radiation therapy & $17(19.8)$ \\
\hline Biological & $4(4.7)$ \\
\hline Other treatment & $5(5.8)$ \\
\hline No previous anticancer treatment & $44(51.2)$ \\
\hline \multicolumn{2}{|l|}{ Planned treatment duration, $n(\%)$} \\
\hline Until progression/death & $27(31.4)$ \\
\hline Fixed number of cycles & $55(64.0)$ \\
\hline Missing & $4(4.7)$ \\
\hline Planned treatment duration (weeks) & $N=51$ \\
\hline Mean \pm SD & $7.7 \pm 3.0$ \\
\hline Min; $\max$ & $1 ; 24$ \\
\hline \multicolumn{2}{|l|}{ Line of treatment, $n(\%)$} \\
\hline Primary & $44(51.1)$ \\
\hline 1st line & $15(17.4)$ \\
\hline 2nd line & $14(16.3)$ \\
\hline 3rd line & $11(12.8)$ \\
\hline Later & $1(1.2)$ \\
\hline Missing & $1(1.2)$ \\
\hline
\end{tabular}

$S D$, standard deviation; $m C R C$, metastatic colorectal cancer; $R / M$, SCCHN metastatic or recurrent squamous cell cancer of the head and neck; $L A S C C H N$, locally advanced squamous cell cancer of the head and neck; $E C O G$, Eastern Cooperative Oncology Group

to continue cetuximab therapy in most patients $(97.7 \%$ in week 2, 93.6\% in week 6 and $93.1 \%$ in week 16). At week 2, 1 patient (1.2\%) strongly favoured continuation of therapy, 
Table 2 Skin reactions by most severe grading in the safety analysis set $(n=102)$

\begin{tabular}{|c|c|c|c|c|c|c|}
\hline Most severe grading & Total, $n(\%)$ & Grade $1, n(\%)$ & Grade $2, n(\%)$ & Grade $3, n(\%)$ & Grade $4, n(\%)$ & Unknown, $n(\%)$ \\
\hline Any skin reaction & $89(87.3)$ & $46(45.1)$ & $38(37.3)$ & $5(4.9)$ & 0 & 0 \\
\hline Rash maculo-papular & $55(53.9)$ & $39(38.2)$ & $12(11.8)$ & $2(2.0)$ & 0 & $2(2.0)$ \\
\hline Rash papulo-pustular & $54(52.9)$ & $30(29.4)$ & $20(19.6)$ & $4(3.9)$ & 0 & 0 \\
\hline Xerosis & $68(66.7)$ & $53(52.0)$ & $12(11.8)$ & 0 & 0 & $3(2.9)$ \\
\hline Pruritus & $34(33.3)$ & $27(26.5)$ & $7(6.9)$ & 0 & 0 & 0 \\
\hline Paronychia & $10(9.8)$ & $8(7.8)$ & $2(2.0)$ & 0 & 0 & 0 \\
\hline Hand-foot skin reactions & $5(4.9)$ & $3(2.9)$ & $2(2.0)$ & 0 & 0 & 0 \\
\hline Other skin reactions & $40(39.2)$ & $30(29.4)$ & $7(6.9)$ & $1(1.0)$ & 0 & $2(2.0)$ \\
\hline
\end{tabular}

increasing to 4 patients $(5.1 \%)$ in week 6 . Furthermore, there was one patient $(1.2 \%)$ at week 2 who strongly favoured discontinuation of therapy.

Patients' perceptions of the measures taken are summarized in Fig. 3. The majority of the patients who reported the perceived measures taken against skin reactions to be neutral reported a neutral effect of skin products on their skin reactions and no change in the skin reactions during the observation period.

\section{Discussion}

This prospective observational study provides a detailed insight into the use, acceptance and the perceived efficacy of measures taken against skin reactions related to cetuximab in the Netherlands. A wide range of skincare products and medications was used in patients with metastatic colorectal cancer (mCRC) and recurrent and/or metastatic or locally advanced squamous cell cancer of head and neck (SCCHN) in 7 sites across the country. The most frequent skin products in the study were moisturizing products, systemic antibiotics and topical antibiotics wherein the use of all products gradually increased during the first 10 weeks of the observation period. Hospital protocols were the primary reason for the choice of skin care and medical products being used as prophylactic or reactive treatment of skin reactions.

These findings are comparable with the results of a similar study conducted in Switzerland, in which patients also received a broad variety of preventive and therapeutic skin care

Table 3 Overall use of the different skin care products

\begin{tabular}{|c|c|c|c|c|c|c|}
\hline Medication/skin care products & Baseline $(N=86)$ & Week $2(N=86)$ & Week $4(N=82)$ & Week $6(N=78)$ & Week $10(N=56)$ & Week $16(N=29)$ \\
\hline \multicolumn{7}{|l|}{ Topical antibiotics } \\
\hline$n(\%)$ & $5(5.8)$ & $24(27.9)$ & $39(47.6)$ & $40(51.3)$ & $25(44.6)$ & $9(31.0)$ \\
\hline $95 \% \mathrm{CI}$ & $1.9-13.0$ & $18.8-38.6$ & $36.4-58.9$ & $39.7-62.8$ & $31.3-58.5$ & $15.3-50.8$ \\
\hline \multicolumn{7}{|l|}{ Systemic antibiotics } \\
\hline$n(\%)$ & $17(19.8)$ & $28(32.6)$ & $39(47.6)$ & $35(44.9)$ & $26(46.4)$ & $8(27.6)$ \\
\hline $95 \% \mathrm{CI}$ & $12.0-29.8$ & $22.8-43.5$ & $36.4-58.9$ & $33.6-56.6$ & $33.0-60.3$ & $12.7-47.2$ \\
\hline \multicolumn{7}{|l|}{ Moisturizing product } \\
\hline$n(\%)$ & $33(38.4)$ & $36(41.9)$ & $41(50.0)$ & $40(51.3)$ & $25(44.6)$ & $10(34.5)$ \\
\hline $95 \% \mathrm{CI}$ & $28.1-49.5$ & $31.3-53.0$ & $38.7-61.3$ & $39.7-62.8$ & $31.3-58.5$ & $17.9-54.3$ \\
\hline \multicolumn{7}{|l|}{ Lipid-regenerating product } \\
\hline$n(\%)$ & $10(11.6)$ & $10(11.6)$ & $18(22.0)$ & $20(25.6)$ & $6(10.7)$ & $1(3.4)$ \\
\hline $95 \% \mathrm{CI}$ & $5.7-20.3$ & $5.7-20.3$ & $13.6-32.5$ & $16.4-36.8$ & $4.0-21.9$ & $0.1-17.8$ \\
\hline \multicolumn{7}{|l|}{ Vitamin $\mathrm{K}_{1}$ cream } \\
\hline$n(\%)$ & $8(9.3)$ & $12(14.0)$ & $11(13.4)$ & $9(11.5)$ & $8(14.3)$ & $5(17.2)$ \\
\hline $95 \% \mathrm{CI}$ & $4.1-17.5$ & $7.4-23.1$ & $6.9-22.7$ & $5.4-20.8$ & $6.4-26.2$ & $5.8-35.8$ \\
\hline \multicolumn{7}{|l|}{ Other topical treatments } \\
\hline$n(\%)$ & 0 & $7(8.1)$ & $14(17.1)$ & $16(20.5)$ & $13(23.2)$ & $3(10.3)$ \\
\hline $95 \% \mathrm{CI}$ & & $3.3-16.1$ & $9.7-27.0$ & $12.2-31.2$ & $13.0-36.4$ & $2.2-27.4$ \\
\hline
\end{tabular}

$C I$, confidence interval 


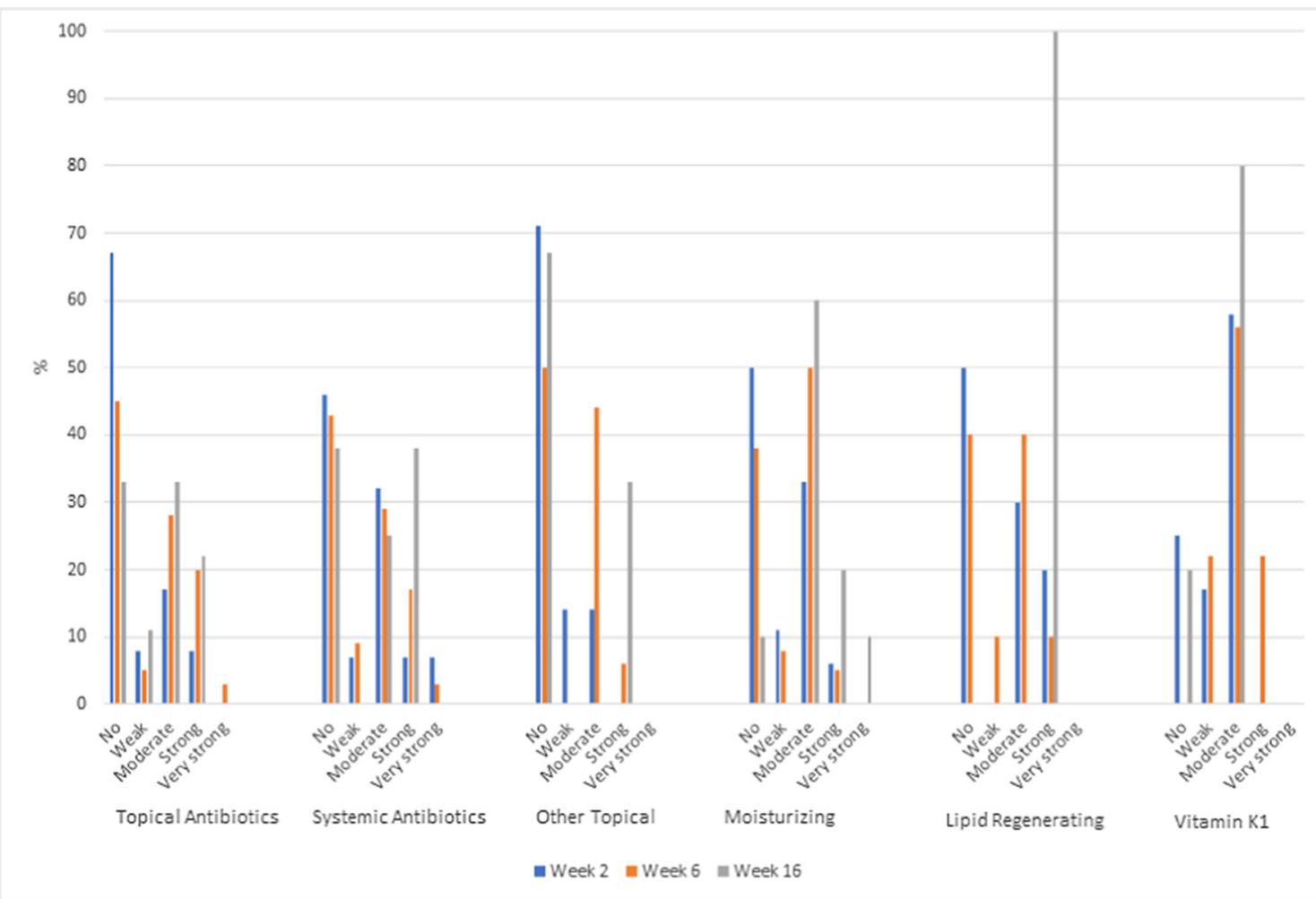

Fig. 2 Healthcare providers' perceived effectiveness of applied skin products

measures for cetuximab-related skin reactions [19]. In the absence of evidence-based guidelines for an optimal approach to prevent and manage cutaneous reactions of EGFR-targeted antibodies, our practice survey and the Swiss study demonstrate the differences in choice of skin care products in the various hospitals and areas. Only a small proportion of patients developed severe cutaneous reactions and for patients, the occurrence of skin reactions did not influence their willingness to continue cetuximab therapy. However, the two studies differ in some ways. In contrast to the Swiss study, the minority of patients in our study was diagnosed with mCRC (30.2\% versus $72.8 \%$ ) and patients were allowed to undergo radiation therapy at the same time. In addition, there was a difference in the choice of skin care products. General skin care products frequently used in Switzerland like ureacontaining products, lipid-regenerating products and vitamin $\mathrm{K}_{1}$ cream were rarely prescribed in the Dutch population. On the other hand, antibiotics (topical and systemic) were more popular in the Netherlands. This can partly be explained by the fact that vitamin $\mathrm{K}_{1}$ cream was not available by prescription in the Netherlands when the study was conducted. In addition, hospital protocols have often been drawn up in consultation with dermatologists, whereas differences in dermatological care between countries are common. Despite these differences in patients and chosen skincare products, the outcome in the studies was in accordance. Remarkably, topical steroids were rarely described in both countries, despite the fact that these agents are often recommended in expert guidelines.

This study was conducted to gain more information about skin reactions related to cetuximab therapy and current measures taken to prevent and manage them in daily practice. It should be mentioned that only 26 out of 102 patients (25.5\%) received monotherapy with cetuximab. 48 patients $(47.1 \%)$ had concurrent radiation therapy, 27 patients $(26.5 \%)$ received a combination of chemotherapy and cetuximab and 1 patient $(1.0 \%)$ was treated with chemotherapy, palliative radiotherapy and cetuximab at the same time. Therefore, not all skin reactions are all necessarily cetuximab-related. There might have been patients with radiation dermatitis or a chemotherapyinduced rash as well. Up to 85 to $95 \%$ of patients treated with radiotherapy developed moderate to severe skin reactions depending on the cumulative radiation dose to the skin [20-22]. Optimal strategies for the prevention and treatment of radiation-induced dermatitis remain a challenge as well with conflicting results in various studies. Washing with water and mild soap, topical corticosteroids and silver nylon dressings have proven to be effective in radiation dermatitis; all strategies were not applied in this survey.

Cutaneous reactions caused by cetuximab can have a serious impact on patients' HRQoL and their willingness to continue treatment [23]. In the present study, no grade 4 skin reactions were observed and less than $5 \%$ of patients had a grade 3 reaction at some point during the observation period. 
Fig. 3 Patients' perceptions of the measures taken and effect on skin reactions

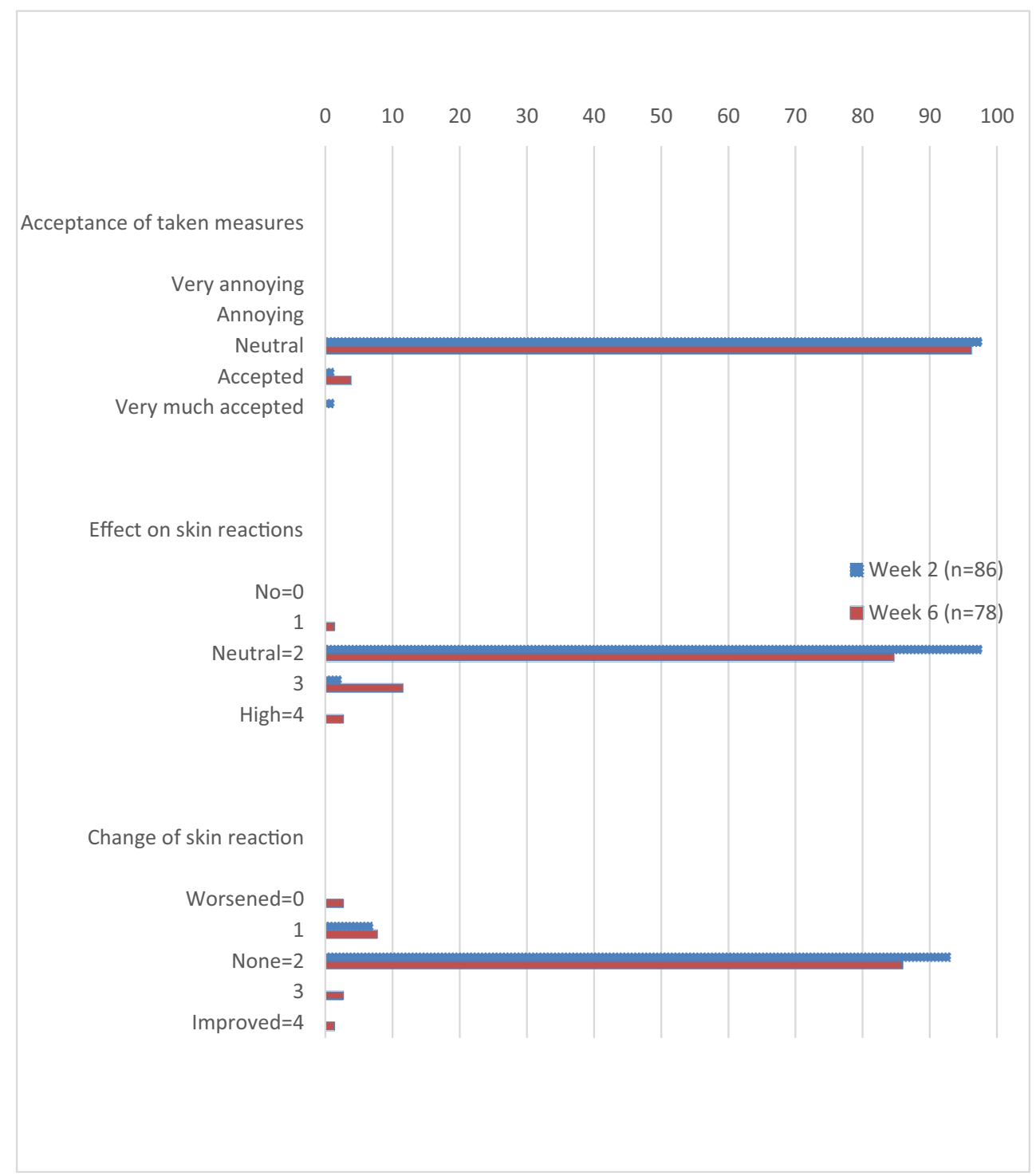

This has ensured that only 1 patient has stopped treatment with cetuximab due to skin reactions. We did see a decrease in the mean HRQoL overall score, as for all domains, from baseline to week 10. Possibly, mild or moderate skin reactions can negatively affect patients' HRQoL and daily life. However, because the majority of patients received radiation or chemotherapy, the declined HRQoL scores cannot only be attributed to cetuximab-related skin reactions. The fact that a large proportion of patients have only been treated for 8 weeks may also have helped the treatment tolerance, as patients can experience a greater negative impact if side effects last longer.

Patients, physicians and nurses perceived the effectiveness of the used products generally comparable for all categories. "No effect" or at most "weak to moderate" effectiveness was reported by the majority of participating patients and physicians. A proportion of patients used multiple products at the same time, like moisturizing products and systemic antibiotics, which made it difficult to distinguish which products were less effective. Patients seemed to be most enthusiastic about moisturizing products, vitamin $\mathrm{K}_{1}$ cream and antibiotics (systemic and topical), whereas healthcare providers reported the highest scores for vitamin $\mathrm{K}_{1}$ cream, lipidregenerating products and systemic antibiotics. The differences between perceived effectiveness assessments were small with no superiority for one of the used agents. In concordance with our Swiss colleagues, we have to conclude that the optimal treatment option needs to be explored individually [19]. Results from previous studies, recommendations and discussions at consensus meetings remain conflicting and so far, no evidence-based guideline for cetuximab-related skin reactions could be established.

There are a number of limitations to this study. Firstly, as a consequence of missing data only 86 patients could be analysed resulting in a relatively small sample size. It was 
the intention to analyse all endpoints from $\mathrm{mCRC}$ and SCCHN patients separately; however, the low number of patients in each subgroup and the high number of skin care medications given precluding meaningful interpretation of data in the subgroups. In addition, it would have been useful to compare the incidence, time of occurrence and grade of skin reactions in patients given prophylactic treatment and those not receiving such treatment. Due to a lack of sufficient data regarding the type of treatment given, this analysis could not be performed in a meaningful way. Secondly, the follow-up period of 16 weeks is relatively short, and many patients discontinued the study early as they were treated for a shorter planned period with cetuximab. Although the development of skin reactions in patients treated with cetuximab usually starts within the first weeks of treatment. Therefore, this time window was chosen for the observation period in this study. Thirdly, the present results were potentially biased in some aspects. Inclusion of patients in a non-blinded observational study with no control arm, at which the treating HCP assigns patients to a certain therapy of their own preference, might have influenced the reported perceived effectiveness. Furthermore, the inclusion of only Dutch patients with access to all available highly qualified care and a good health insurance system might limit the generalizability of these findings to patients in lower- or middle-income countries.

\section{Conclusions}

Our study shows that Dutch oncologists and nurses used a variety of products for the prevention or treatment of cetuximab-induced skin reaction, mostly based on the local hospital protocols. In general, both patients and treating healthcare providers perceived at most moderate efficacy of the various measures. Fortunately, the skin reactions that occurred during treatment were mostly mild or moderate, had no large impact on daily life and did not lead to discontinuation of treatment.

Acknowledgements We thank all patients who participated in the Proskin study. In addition, we also thank M. Soesan from the Slotervaart Hospital (Amsterdam, The Netherlands) and all involved research nurses for their contributions.

Author contributions Commissioned by Merck KGaA, Christine BoersDoets designed and wrote the Dutch study protocol, based on the Swiss protocol, and collected data.

Material preparation, data collection and analysis were performed by MakroCare Clinical Research Limited, India.

The first draft of the manuscript was written by Rieneke Lugtenberg and Hans Gelderblom and all authors commented on previous versions of the manuscript.

Funding This study was sponsored by Merck B.V., Schiphol-Rijk, The Netherlands, an affiliate of Merck KGaA, Darmstadt, Germany.
Data availability The datasets generated and/or analysed during the current study are not publicly available as sharing is not explicitly covered by patient consent.

\section{Compliance with ethical standards}

Conflict of interest This study was sponsored by Merck B.V., SchipholRijk, The Netherlands, an affiliate of Merck KGaA, Darmstadt, Germany. C. del Grande and B. van Doorn are employees of Merck B.V. The other authors do not have financial relationships with Merck B.V.

Ethics approval This study was conducted in accordance with the protocol, the ethical principles laid down in the Declaration of Helsinki, International Council for Harmonization of Technical Requirements for Pharmaceuticals for Human Use and Good Clinical Practice (ICH GCP), guidelines and applicable regulatory requirements. The protocol, ICF, any other materials provided to the patients and further requested information were submitted to the IEC/IRB and the competent authority. The regulatory application or submission for regulatory approval was made by the sponsor or designee as required by national law. The competent authority and the IEC/IRB were notified of the end of the study in accordance with local regulations.

Consent to participate Informed consent was obtained from all individual participants included in the study.

Consent for publication All authors read and approved the final manuscript.

Open Access This article is licensed under a Creative Commons Attribution 4.0 International License, which permits use, sharing, adaptation, distribution and reproduction in any medium or format, as long as you give appropriate credit to the original author(s) and the source, provide a link to the Creative Commons licence, and indicate if changes were made. The images or other third party material in this article are included in the article's Creative Commons licence, unless indicated otherwise in a credit line to the material. If material is not included in the article's Creative Commons licence and your intended use is not permitted by statutory regulation or exceeds the permitted use, you will need to obtain permission directly from the copyright holder. To view a copy of this licence, visit http://creativecommons.org/licenses/by/4.0/.

\section{References}

1. Bonner JA, Harari PM, Giralt J, Azarnia N, Shin DM, Cohen RB, Jones CU, Sur R, Raben D, Jassem J, Ove R, Kies MS, Baselga J, Youssoufian H, Amellal N, Rowinsky EK, Ang KK (2006) Radiotherapy plus cetuximab for squamous-cell carcinoma of the head and neck. N Engl J Med 354(6):567-578

2. Jonker DJ, O'Callaghan CJ, Karapetis CS, Zalcberg JR, Tu D, Au $\mathrm{HJ}$ et al (2007) Cetuximab for the treatment of colorectal cancer. N Engl J Med 357(20):2040-2048

3. Karapetis CS, Khambata-Ford S, Jonker DJ, O'Callaghan CJ, Tu $\mathrm{D}$, Tebbutt NC et al (2008) K-ras mutations and benefit from cetuximab in advanced colorectal cancer. N Engl J Med 359(17): $1757-1765$

4. Vermorken JB, Mesia R, Rivera F, Remenar E, Kawecki A, Rottey S, Erfan J, Zabolotnyy D, Kienzer HR, Cupissol D, Peyrade F, Benasso M, Vynnychenko I, de Raucourt D, Bokemeyer C, Schueler A, Amellal N, Hitt R (2008) Platinum-based 
chemotherapy plus cetuximab in head and neck cancer. N Engl J Med 359(11):1116-1127

5. Galimont-Collen AF, Vos LE, Lavrijsen AP, Ouwerkerk J, Gelderblom H (2007) Classification and management of skin, hair, nail and mucosal side-effects of epidermal growth factor receptor (EGFR) inhibitors. Eur J Cancer 43(5):845-851

6. Lacouture ME (2006) Mechanisms of cutaneous toxicities to EGFR inhibitors. Nat Rev Cancer 6(10):803-812

7. De Tursi M, Zilli M, Carella C, Auriemma M, Lisco MN, Di Nicola $M$ et al (2017) Skin toxicity evaluation in patients treated with cetuximab for metastatic colorectal cancer: a new tool for more accurate comprehension of quality of life impacts. Onco Targets Ther 10:3007-3015

8. Joshi SS, Ortiz S, Witherspoon JN, Rademaker A, West DP, Anderson R, Rosenbaum SE, Lacouture ME (2010) Effects of epidermal growth factor receptor inhibitor-induced dermatologic toxicities on quality of life. Cancer. 116(16):3916-3923

9. Holch JW, Held S, Stintzing S, Fischer von Weikersthal L, Decker $\mathrm{T}$, Kiani A et al (2020) Relation of cetuximab-induced skin toxicity and early tumor shrinkage in metastatic colorectal cancer patients: results of the randomized phase 3 trial FIRE-3 (AIO KRK0306). Ann Oncol Off J Eur Soc Med Oncol 31(1):72-78

10. Stintzing S, Kapaun C, Laubender RP, Jung A, Neumann J, Modest DP, Giessen C, Moosmann N, Wollenberg A, Kirchner T, Heinemann V (2013) Prognostic value of cetuximab-related skin toxicity in metastatic colorectal cancer patients and its correlation with parameters of the epidermal growth factor receptor signal transduction pathway: results from a randomized trial of the GERMAN AIO CRC Study Group. Int J Cancer 132(1):236-245

11. Uozumi S, Enokida T, Suzuki S, Nishizawa A, Kamata H, Okano T, Fujisawa T, Ueda Y, Okano S, Tahara M, Yamaguchi M (2018) Predictive value of cetuximab-induced skin toxicity in recurrent or metastatic squamous cell carcinoma of the head and NECK. Front Oncol 8:616

12. Brown J, Su Y, Nelleson D, Shankar P, Mayo C (2016) Management of epidermal growth factor receptor inhibitorassociated rash: a systematic review. J Commun Support Oncol 14(1):21-28

13. Lacouture ME, Anadkat MJ, Bensadoun R-J, Bryce J, Chan A, Epstein JB et al (2011) Clinical practice guidelines for the prevention and treatmentof EGFR inhibitor-associated dermatologic toxicities. Support Care Cancer 19(8):1079-95

14. Scope A, Agero AL, Dusza SW, Myskowski PL, Lieb JA, Saltz L et al (2007) Randomized double-blind trial of prophylactic oral minocycline and topical tazarotene for cetuximab-associated acnelike eruption. J Clin Oncol 25(34):5390-5396

15. Baas JM, Krens LL, Guchelaar HJ, Ouwerkerk J, de Jong FA, Lavrijsen AP et al (2012) Recommendations on management of EGFR inhibitor-induced skin toxicity: a systematic review. Cancer Treat Rev 38(5):505-514

16. Hofheinz RD, Lorenzen S, Trojan J, Ocvirk J, Ettrich TJ, Al-Batran SE et al (2018) EVITA-a double-blind, vehicle-controlled, randomized phase II trial of vitamin $\mathrm{K}_{1}$ cream as prophylaxis for cetuximab-induced skin toxicity. Ann Oncol Off J Eur Soc Med Oncol 29(4):1010-1015

17. Boers-Doets CB, Gelderblom H, Lacouture ME, Epstein JB, Nortier JW, Kaptein AA (2013) Experiences with the FACTEGFRI-18 instrument in EGFRI-associated mucocutaneous adverse events. Suppor Care Cancer Off J Multinatl Assoc Support Care Cancer 21(7):1919-1926

18. Wagner LI, Berg SR, Gandhi M, Hlubocky FJ, Webster K, Aneja M, Cella D, Lacouture ME (2013) The development of a Functional Assessment of Cancer Therapy (FACT) questionnaire to assess dermatologic symptoms associated with epidermal growth factor receptor inhibitors (FACT-EGFRI-18). Suppor Care Cancer Off J Multinatl Assoc Support Care Cancer 21(4):1033-1041

19. Rothschild SI, Betticher D, Zenhausern R, Anchisi S, von Moos R, Pless M et al (2019) Prospective, observational practice survey of applied skin care and management of cetuximab-related skin reactions: PROSKIN study. Cancer Chemother Pharmacol 84(4):881889

20. Hegedus F, Mathew LM, Schwartz RA (2017) Radiation dermatitis: an overview. Int J Dermatol 56(9):909-914

21. Rosenthal A, Israilevich R, Moy R (2019) Management of acute radiation dermatitis: a review of the literature and proposal for treatment algorithm. J Am Acad Dermatol 81(2):558-567

22. Koldenhof JJ, Langenberg MHG, Witteveen PO, Teunissen S (2018) Patient-reported symptoms and stepwise symptom management in patients on epidermal growth factor inhibitors: a retrospective, descriptive cohort study. Eur J Cancer Care 27(2):e12800

23. Chiang TY, Hsu HC, Jane SW, Chen SC (2020) EGFRI-associated health-related quality of life by severity of skin toxicity in metastatic colorectal cancer patients receiving epidermal growth factor receptor inhibitor target therapy. Suppor Care Cancer Off J Multinatl Assoc Support Care Cancer 28:4771-4779

Publisher's note Springer Nature remains neutral with regard to jurisdictional claims in published maps and institutional affiliations. 